UCRL-ID-125491

\title{
Status Report on Corsica Modeling for Current Drive Scenario Development
}

\author{
T.A. Casper, J. Crotinger, J.M. Moller, L.D. Pearlstein
}

\section{RECEIVED \\ JAN 171997 \\ OSTI}

\section{NSTRENTW OF THS DCOUMET IS UMLMITED

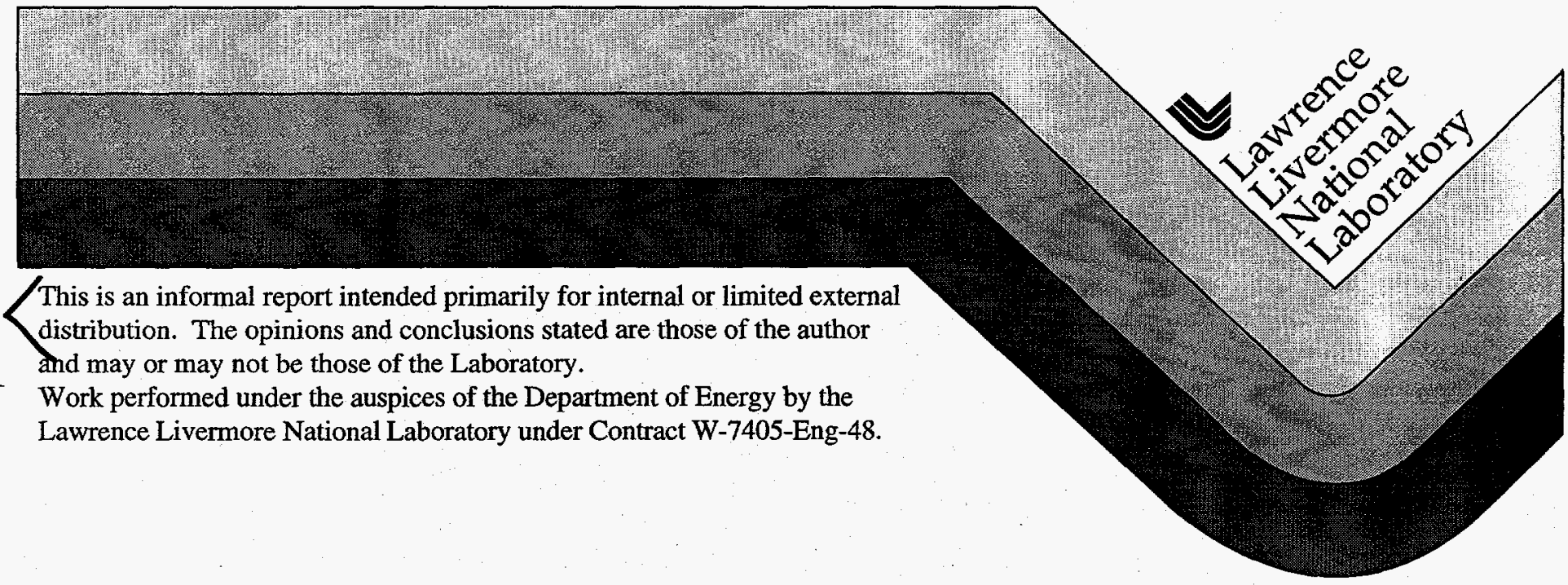




\section{DISCLAIMER}

This document was prepared as an account of work sponsored by an agency of the United States Government. Neither the United States Government nor the University of California nor any of their employees, makes any warranty, express or implied, or assumes any legal liability or responsibility for the accuracy, completeness, or usefulness of any information, apparatus, product, or process disclosed, or represents that its use would not infringe privately owned rights. Reference herein to any specific commercial product, process, or service by trade name, trademark, manufacturer, or otherwise, does not necessarily constitute or imply its endorsement, recommendation, or favoring by the United States Government or the University of California. The views and opinions of authors expressed herein do not necessarily state or reflect those of the United States Government or the University of California, and shall not be used for advertising or product endorsement purposes.

This report has been reproduced directly from the best available copy.

Available to DOE and DOE contractors from the Office of Scientific and Technical Information P.O. Box 62, Oak Ridge, TN 37831

Prices available from (615) 576-8401, FTS 626-8401

Available to the public from the

National Technical Information Service

U.S. Department of Commerce

5285 Port Royal Rd.

Springfield, VA 2216 


\section{DISCLAMMER}

Portions of this document may be illegible in electronic image products. Images are produced from the best available original document. 


\title{
Status report on Corsica modeling for current drive scenario development
}

\author{
T.A. Casper, J. Crotinger, J.M. Moller, L.D. Pearlstein \\ September 30, 1996
}

\section{SUMMARY}

This milestone report covers the progress and status of Corsica modeling for DIII-D experiments over the past year, since our previous report in September, 1995. During this time, we have concentrated on improvements to the code in support of our ability to do self-consistent, predictive modeling of DIII-D discharges. Our interest is in obtaining a tool, benchmarked with experimental data, for developing advanced tokamak operations scenarios including simulation and analysis of high performance negative central shear (NCS) discharges and control of the current profile evolution. Our major focus has been on installing and improving the neutral beam current drive model in Corsica; this element is critical to modeling the evolution of DIII-D discharges. The NFREYA ${ }^{1}$ neutral beam deposition code was installed (starting with a version consistent with GA's ONETWO code) and the capability for following particle orbits, including the effects of drifts, was added for determining the current driven by neutral beam injection. In addition, improved methods for more easily integrating experimental profile measurements into the code operation and for calculating $Z_{\text {eff }}$ either from models or from impurity density measurements have been added. We have recently begun to turn on various transport models in our simulation of discharge evolution. We have concentrated on the NCS configuration and have simulated the evolution of two different high neutron reactivity discharges; an NCS discharge with L-mode edge ${ }^{2}$ and a single-null, weak NCS discharge from the JET/ITER/DIII-D ${ }^{3}$ equivalent shape experiments. Corsica simulation ${ }^{4}$ results for these discharges were presented at the EPS meeting in Kiev, Ukraine in June, 1996.

\subsection{INTRODUCTION}

Corsica $^{5}$ is a time-dependent equilibrium and transport code that was formulated and developed mostly under internal funding at LLNL. It couples 1-D, slow-timescale core transport to 2-D, free-boundary MHD equilibrium including the effects of external coil currents. The core processes have also been coupled to the edge physics code UEDGE ${ }^{6}$. Free and fixed boundary equilibrium calculation options are available with the inclusion of currents flowing in poloidal field coils and in passive wall structures. GA's EFIT polynomial representation for the pressure and current profile functions, $\mathrm{p}^{\prime}$

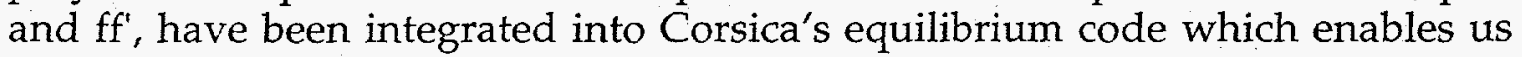


to reproduce EFIT equilibria. In addition, Corsica provides for the calculation of Mercier, ballooning, and vertical stability during modeling of the discharge evolution.

While development of code structure and initial implementation have been completed under LLNL internal funding, new physics models have been incorporated as part of the LLNL theory and experimental efforts. A significant effort from the LLNL theory group was required for the implementation of the NFREYA code, particularly in the development of the particle orbit code for determining the spatial distribution of current drive using the particle transit time in flux zones. Simulation of the evolution of DIII-D discharges, code comparisons with experimental data, and some programming support were completed as part of LLNL's collaboration on the DIII-D experiment.

\subsection{APPLICATION TO NCS MODELING EFFORTS}

Our current modeling with the Corsica code is directed at establishing the ability to accurately simulate the evolution of the high performance NCS discharges. Before proceeding with predictive modeling appropriated for current profile control, we must insure that we have the ability to qualitatively and quantitatively simulate features present in the DIII-D discharges. We have explored two cases of current LLNL programmatic interest in the advance tokamak (AT) effort, both are high performance NCS discharges. The NCS configuration has emerged as a confinement regime exhibiting high neutron reactivity and promise for future advanced tokamak modes of operation. Some of the highest neutron rates achieved in DIII-D experiments have been obtained in the NCS configuration. Due to it's advantage as an attractive AT scenario, we are simulating this configuration to explore issues of extending performance to longer duration in DIII-D and to steady state for future experiments.

We have simulated portions of two DIII-D discharges with distinctly different features:

1. Shot 84682: double-null, NCS with a peaked pressure profile and L-mode edge. We have simulated the evolution of a transition from the early neutral beam injection phase used to form the NCS q-profile to the L-mode-edge high performance phase.

2. Shot 88964: single null, NCS in the JET/DIII-D/ITER equivalent shape with an $\mathrm{H}$-mode-like (broad) pressure profile. We have simulated the transition to high performance during the $\mathrm{H}$-mode-like phase of this discharge.

Simulations of these two discharges were summarized and presented at the EPS $^{4}$ meeting by T. A. Casper, et. al.. A portion of the paper to be published in the EPS proceedings is included here: 


\section{Simulation methodology}

We are presently simulating the temporal evolution of the current profiles to develop an understanding and validation of the models for forming and sustaining the high performance NCS configuration. At this time, we use experimental measurements of the density and temperature profiles rather than model the particle and energy transport. We take as input the measured electron and impurity densities and the electron and ion temperatures and infer the ion density and effective charge $\left(Z_{\text {eff }}\right)$ from quasineutrality using carbon as the main impurity. We initialize the equilibrium by choosing the pressure ( $\mathrm{p}^{\prime}$ ) and current (ff') parameterizations used in fitting data with EFIT. A free boundary calculation provides a starting equilibrium with forced convergence to the plasma current and fitted values of the F-coil currents to account for currents in structures. This prescription allows us to directly compare with the EFIT results and boundary shapes produced in this manner are in generally good agreement. The simulated discharge is evolved from this initial state using a fixed boundary equilibrium calculation while simultaneously accounting for current diffusion.

In previous simulations, an approximate particle orbit model resulted in too little neutral beam current drive on axis. We have replaced this portion of our neutral beam injection code with an orbit following calculation for the Monte Carlo simulation of neutral beam current drive. The injection process is now tightly coupled to the local equilibrium flux surfaces with current drive determined from the residence time of particles in flux zones. By evaluating the trapped, passing, and lost particle distributions we infer the direct current drive from passing beam particle orbits and the bootstrap contribution due to the trapped injected ions.

\section{Simulation Results}

We have begun our modeling with two different high performance discharges. Shot 84682 is a double null, negative central shear discharge with L-mode-like peaked pressure profiles ${ }^{2}$. Confinement factors up to $\mathrm{H} 2.5$ were obtained while maintaining an L-mode edge pressure profile which allows for good penetration of the neutral beams. The inverted q-profile is achieved by early neutral beam injection during the Ohmic current ramp-up phase of the experiment. Ion temperatures in excess of $15 \mathrm{KeV}$ were obtained with peaked density profiles, $n(0) /<n>\sim 2.2$. A second case, shot 88964 , is a single null, weak central shear discharge with $\mathrm{H}$-mode-like pressure profiles giving a broader deposition profile for the neutral beam injection. The weak shear is formed at reduced neutral beam injection power during the Ohmic ramp. This DIII-D shape is representative of the JET and ITER advanced tokamak scenarios ${ }^{3,7}$. 
In Figure 1, we show the plasma current and neutral beam injection histories for these two shots along with the simulation time interval. We model the neutral beam injection as two aggregate sources having the proper geometry for the two beamline orientations on DIII-D and step the average

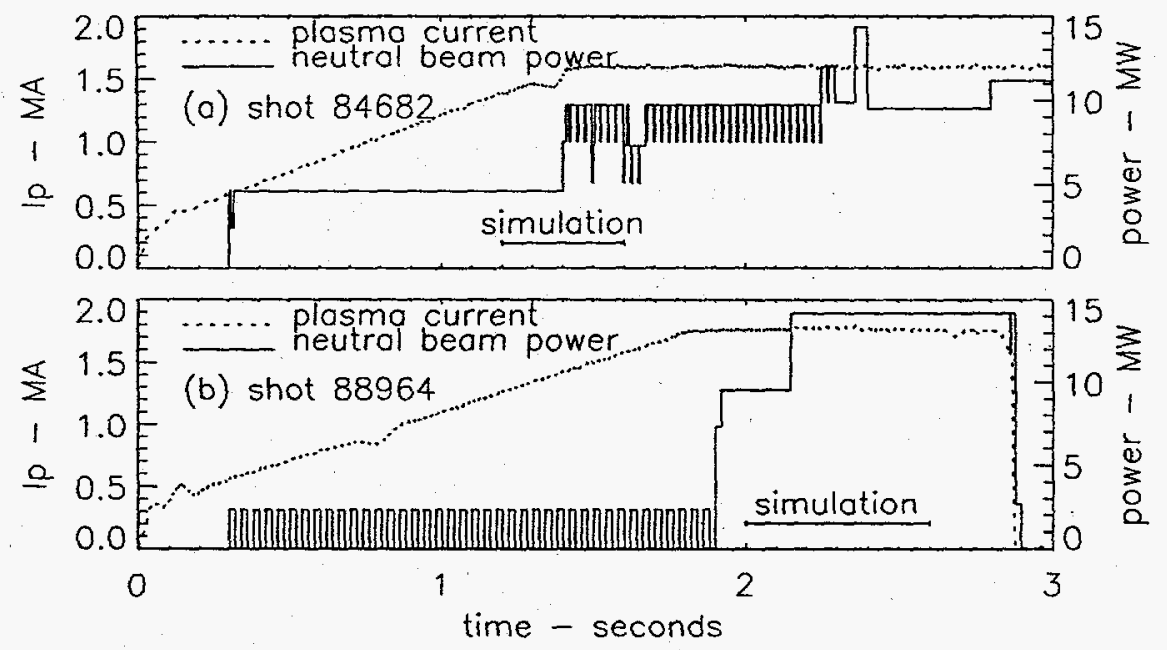

Figure 1. Time history of plasma current and neutral beam power injected.

power in time consistent with the experimental variations. Corsica is run in time dependent mode to simulate the evolution of the equilibrium associated with the current diffusion process and obtain spatial-temporal profiles of plasma parameters. We show profiles of the total, Ohmic, bootstrap, neutral beam driven densities in Figure 2 at times near the peak stored energy.
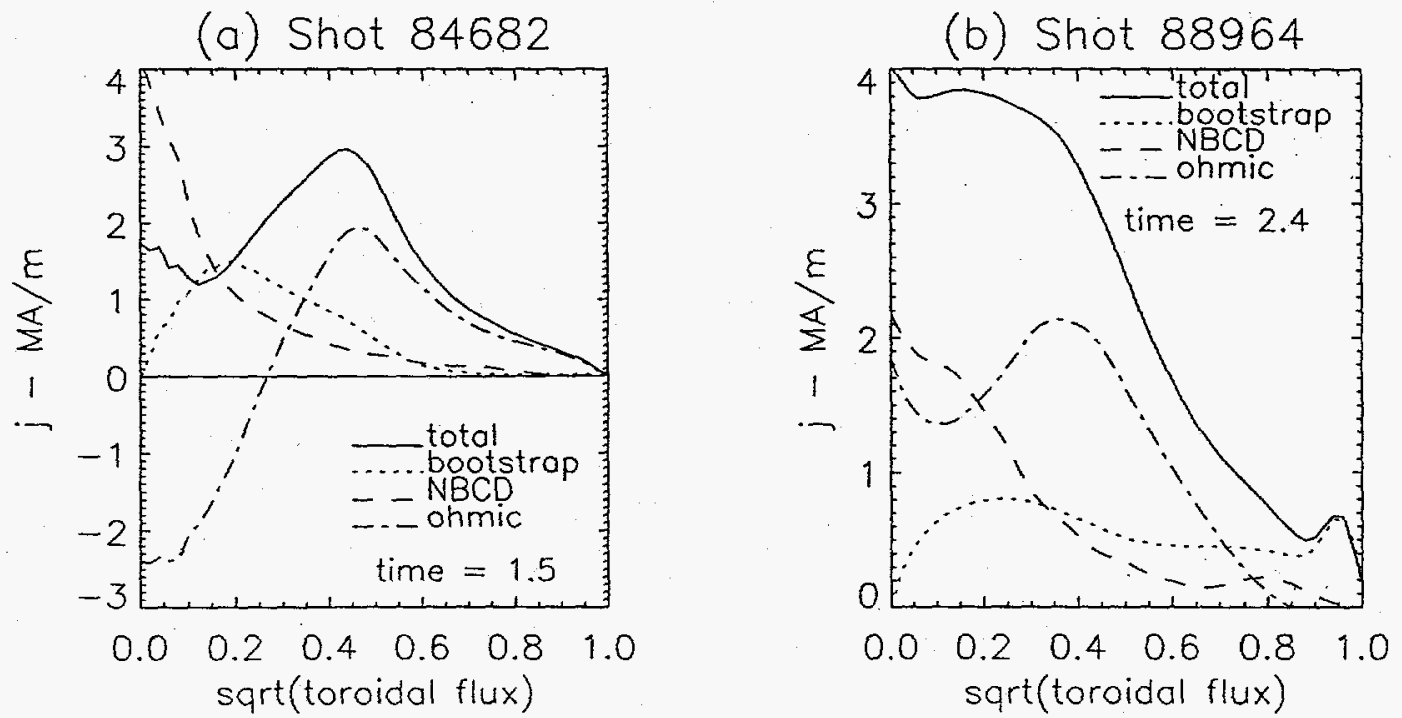

Figure 2. Current density profiles at times near peak stored energy 
As indicated in Figure 3a for the NCS simulation (84682), we obtain good agreement between the simulated q-profile evolution and EFIT analysis outside of $\rho=0.2(\rho=\sqrt{ }($ toroidal flux $))$, where we note good agreement with the minimum value of $q$ and of $q_{95}$. Inside $\rho=0.2$, the current drive due to neutral beam injection dominates the total current and forces the simulation $\mathrm{q}_{0}$ to drop considerably below that inferred from the EFIT reconstruction. For the weak shear simulation case, Figure $3 b$, there is agreement over most of the profile during the entire simulation. However, the simulated q-profile evolution does not see the weak bump near $\rho \sim 0.3$ that arises in the EFIT analysis during the times that the density and temperature and, presumably, the ensuing current distribution are rapidly changing. The presence of this bump is critically dependent on the fit parameters used in the analysis. We are currently investigating the details of the current drive associated with the neutral beam injection which dominates these and all high performance discharges in DIII-D.

(a) Shot 84682

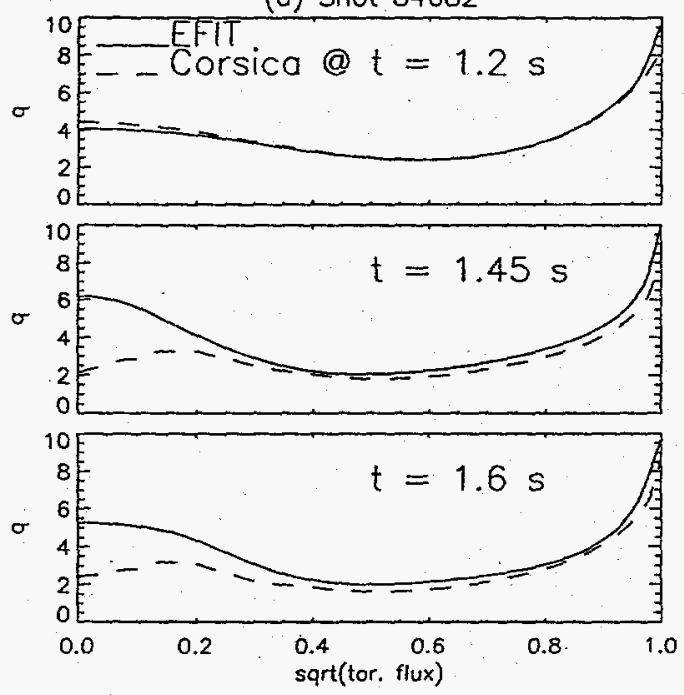

(b) Shot 88964

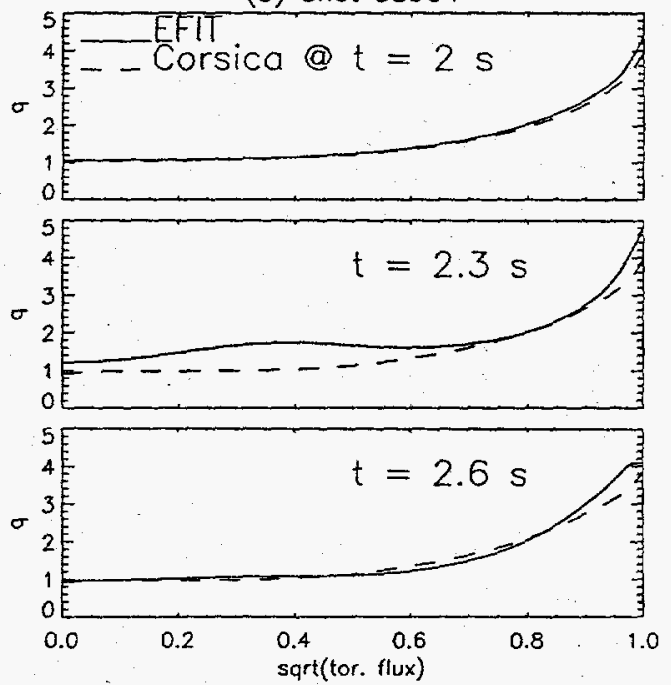

Figure 3. Simulation q-profiles with data fits from EFIT for comparison.

In summary, the initial results at simulating the formation of high performance discharges in DIII-D using our new neutral beam current drive calculation in a fully predictive simulation code are encouraging. We are able to simulate the characteristics of the current profile formation during the evolution of these difficult, high performance discharges and we are in general agreement with the experimental data fitting. Discrepancies due to the peaking of the neutral beam current drive near the magnetic axis are a concern and we are investigating the details of these differences. 


\subsection{CODE IMPROVEMENTS}

\section{Orbit calculation and current drive}

A major addition to the Corsica code is the ability to calculate the neutral beam current drive from the injected particle distribution. The NFREYA code uses a Monte Carlo technique to deposit neutral beam power and density over the plasma cross section. The DIII-D beamline geometry and the injector characteristics are implemented which provides a $2 \mathrm{D}$ description of the deposited ion distribution. To this result, we have added an orbit calculation to determine the fraction of time a particle spends in a given poloidal flux zone. The transit time in each flux zone then provides a measure of the local current drive distribution, including the calculation of trapped, passing, and lost particle orbits. In Figure 4a we show a typical diagnostic plot indicative of typical banana orbits obtained with conditions representative of DIII-D NCS discharges. We also show the normalized time that a particle spends in a flux zone, Figure $4 \mathrm{~b}$, as a function of the normalized flux and the weighting applied to the current drive calculation in Figure $4 \mathrm{c}$. From this data we obtain the spatial dependence of the neutral beam current drive.

During the benchmarking process, we found the neutral-beam-driven current profile resulting from our initial implementation (NFREYA plus orbit effects) was too highly peaked on axis. This pronounced on-axis peaking, a result of the orbit following calculation, causes a depression in $q_{0}$ which is inconsistent with the measurements on DIII-D. This effect is localized to a region near the magnetic axis as was discussed earlier. Since these results were obtained and largely motivated by them, we have continued to improve our modeling in this area by the addition of particle drifts to the calculation of the orbit time. The weighting of the beam density onto flux surfaces is proportional to the relative time spent on flux surfaces and the addition of the drifts was critical for the determination of the spatial dependence of the neutral beam current drive. These additions have been incorporated in the code and are giving broader current drive distributions. The process of comparing the result of these new additions to the available experimental data are currently on-going and will be discussed in a future report. We are now ready to move these subroutines back to GA to be incorporated into the ONETWO transport analysis code.

\section{Experimental Profiles}

We have made improvements in our capability to input and use experimental profiles in Corsica. Corsica, designed to allow considerable flexibility in the manner in which it can be run and with the interpretive BASIS environment, makes a fairly versatile simulation environment. 


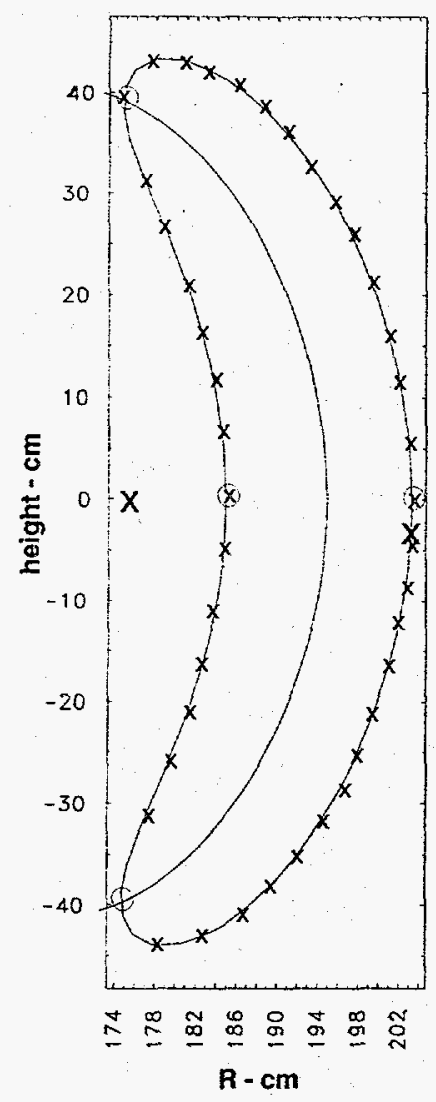

(a) trapped orbit


Figure 4. Diagnostic output from orbit following code for banana orbit

We generally run Corsica in a hybrid simulation mode where available experimental measurements can be used to give the best description of certain features; density, temperature, impurity, and/or $Z_{\text {eff }}$ profiles, that we do not necessarily wish to simulate. Alternatively, we may want to have readily 
accessible any available measurements to facilitate our comparison with the experimental data.

In a parallel effort at LLNL to apply Corsica modeling to the TFTR experiment $t^{8}$, a method was developed to input experimental profiles from UFILEs and create a data base internal to BASIS from which the data can be used. We have adopted this structure for use in our DIII-D modeling effort and now form similar shot-dependent profile data bases in the BASIS/Corsica environment. UFILEs, such as those from PPPL's TRANSP code or GA's analysis code $4 \mathrm{D}$, can now be saved in the data base and read into Corsica for easy comparisons with experimental profiles or for use in simulating the evolution of a discharge.

\subsection{FUTURE DIRECTIONS}

We are presently verifying the ability of Corsica to predictively evolve the current distribution (Ohmic, bootstrap, and neutral beam) subject to neoclassical conductivity for the NCS discharges. These features include the major current drive sources presently available on the DIII-D experiment for modifying the q-profile and other off-axis current drive sources (i.e. ECH or mode conversion $\mathrm{ICH}$ ) will be added when appropriate for modeling future scenarios on DIII-D. We are using measured density and temperature profiles for electrons and ions and include the $Z_{\text {eff }}$ effects using either a model of $Z_{\text {eff }}$ or the carbon impurity measurements with quasineutrality. Using measured profiles circumvents the need to know the exact form for the transport.

To provide predictive capability, however, some assumption on the transport characteristics must be made to be able to investigate methods for control and for finding steady-state configurations. For this reason, we have begun to use transport models. The transport models currently installed in Corsica are: a polynomial diffusion coefficient model, Chang-Hinton ${ }^{9}$ neoclassical model, $\mathrm{RLW}^{10}$, and a first principles model by Kotschenreuther ${ }^{11}$, et. al., is available with a similar model by Waltz ${ }^{12}$, et. al., nearly installed. Initial simulations aimed solely at getting the various models operational have been completed. A collaboration with the University of California at Berkeley was recently begun with the addition of a student working in the area of DIII-D transport modeling using the Corsica code. This will be a major direction for our Corsica simulation efforts over the next year.

\subsection{REFERENCES}

1. Lister, G.G., et. al., Third Symposium on Plasma Heating in Toroidal Devices, Varenna, Italy, 1976. and Fowler, R.H., et. al., ORNL/TM-6845, July, 1979.

2. Rice, B.W., et. al., Phys. Plasmas 3, 1983 (1996). 
3. Strait, E.J., et. al., to be published, Proceedings 23rd European Physical Society Conference on Controlled Fusion and Plasma Physics, Kiev, Ukraine, June, 1996.

4. Casper, T.A., et. al., to be published, Proceedings 23rd European Physical Society Conference on Controlled Fusion and Plasma Physics, Kiev, Ukraine, June, 1996.

5. Crotinger, J. et. al., Bull. Am. Phys. Soc. 382016 (1993).

6. Tarditi, A., et. al., Contributions to Plasma Physics, 36, 132 (1996).

7. Nevins, W. M., et. al. IAEA-CN-60/E-P5, 543, Seville, 1994.

8. Hooper, E.B., et. al., UCRL-ID-124818, July 18, 1996.

9. Chang, C.S., et. al., Phys. of Fluids 29, 3314 (1986).

10. Rebut, P.H. et. al., Phys. of Fluids B3, 2209 (1991).

11. Kotschenreuther, M., et. al., Plasma Physics, 2, 2381 (1995).

12. Waltz, R.E., et.al. Bull. Am. Phys. Soc. 40, 1733 (1995). 